Itinéraires Itinéraires

Littérature, textes, cultures

\title{
Tensions entre les avant-gardes : le surréalisme et le Parti communiste
}

\section{Guillaume Bridet}

\section{(2) OpenEdition}

\section{Journals}

Édition électronique

URL : http://journals.openedition.org/itineraires/1366

DOI : $10.4000 /$ itineraires. 1366

ISSN : 2427-920X

Éditeur

Pléiade

\section{Édition imprimée}

Date de publication : 1 décembre 2011

Pagination : 23-45

ISBN : 978-2-296-55744-4

ISSN : $2100-1340$

\section{Référence électronique}

Guillaume Bridet, «Tensions entre les avant-gardes : le surréalisme et le Parti communiste », Itinéraires [En ligne], 2011-4 | 2011, mis en ligne le 01 décembre 2011, consulté le 30 avril 2019. URL : http:// journals.openedition.org/itineraires/1366; DOI : 10.4000/itineraires.1366

\section{(ब) $(\Theta \Theta$}

Itinéraires est mis à disposition selon les termes de la licence Creative Commons Attribution - Pas d'Utilisation Commerciale - Pas de Modification 4.0 International. 


\title{
Tensions entre les avant-gardes : le surréalisme et le Parti communiste
}

\begin{abstract}
During ten years, the Surrealist group and the Communist Party attempt to get closer to each other, but with no success and, paradoxacally, due to their common beliefs. They both adhere to the avant-garde and share the same desire to destroy capitalist society and bourgeois values. Whereas the Surrealist group belongs to the poetic avantgarde, the Communist Party to that of the political one. Both consider their point of view superior to the other. Their priorities are not the same and their members think, act and live differently.
\end{abstract}

Keywords : surrealism, communist party, Clarté Movement, avant-garde, ethos Mots clés : surréalisme, parti communiste, mouvement Clarté, avant-garde, éthos

Le but de cet article n'est pas de faire l'histoire (tumultueuse) des rapports entre le groupe surréaliste et le Parti communiste français en plaçant le point d'observation d'un côté plutôt que de l'autre, ni et encore moins de suivre les trajectoires individuelles qui ont pu conduire certains membres du groupe à s'inscrire au Parti pour y demeurer longuement ou au contraire pour s'en retirer bien vite. Aussi bien, des ouvrages biographiques ont déjà apporté des éléments de réponse concernant le parcours de tel ou tel poète ou artiste du groupe surréaliste et on trouve dans quelques ouvrages plus généraux des éléments qui, que ce soit dans le domaine des études littéraires, de l'histoire ou de la sociologie, composent l'histoire politique collective du mouvement ou l'histoire du Parti communiste dans son rapport avec les écrivains et les artistes surréalistes. Si nous ne nous interdirons pas de puiser des faits et des analyses dans certains de ces ouvrages - en particulier ceux, devenus classiques, de David Caute, Jean-Pierre A. Bernard, Jean-Pierre Morel ou Carole Reynaud Paligot -, notre projet est toutefois différent : il s'agit d'approcher la rencontre entre le groupe surréaliste et le Parti communiste français en installant le point de vue au lieu même de leur convergence et de leur divergence, 
$i$. e. en nous efforçant de comprendre les raisons profondes qui, de part et d'autre, ont conduit à un rapprochement finalement intenable du fait même de deux positionnements comparables dans leur champ respectif. Comment les deux ensembles sont-ils organisés (point de vue institutionnel)? Quels discours légitiment leur organisation et leur action (point de vue idéologique)? Quels buts visent-ils à long terme (point de vue stratégique) et comment l'adaptent-ils à une certaine conjoncture (point de vue tactique)? Il s'agit de dégager les points communs et les antagonismes qui sont à l'œuvre entre les différents registres d'organisation, de pensée et d'action mobilisés de part et d'autre par un groupe littéraire et par un parti politique qui - point décisif - revendiquent l'un comme l'autre une position avant-gardiste et qui soumettent donc l'individu à une contrainte collective, inscrivent leur action dans le sens de l'histoire et aspirent à transformer radicalement l'homme et la société. Le Parti communiste et le groupe surréaliste apparaissent de ce point de vue et de manière éminemment paradoxale séparés autant que liés par ce qui les rapproche : dépositaires tous deux de projets révolutionnaires totalisants qui ont vocation à se rencontrer mais dont l'un se déploie à partir de l'ordre littéraire, tandis que l'autre s'étend à partir de l'ordre politique - ce qui ne peut manquer de générer, non pas de la rivalité à proprement parler (les deux institutions n'appartiennent pas au même champ), mais de l'incompréhension (elles occupent dans leur champ respectif deux positions homologues mais programmant chacune un éthos et conduisant à des prises de position qui se révèlent incompatibles).

Pour saisir au mieux la rencontre du mouvement surréaliste et du Parti communiste, il faut commencer par considérer ces deux ensembles comme des institutions, c'est-à-dire des groupes plus ou moins importants mais durablement organisés et obéissant à un certain type de fonctionnement. La première chose qui vient à l'esprit sous ce rapport, c'est à la fois leur nature très différente et leur poids très inégal. D'un côté, on a un parti politique composé de militants, de l'autre, un groupe d'écrivains et d'artistes; d'un côté, un regroupement de plusieurs dizaines à quelques centaines de milliers de membres (dans les années 1920 et 1930); de l'autre, quelques dizaines d'individus; d'un côté donc, des ressources humaines, logistiques et financières considérables (que l'aide de l'URSS contribue encore à amplifier), de l'autre, des possibilités d'action bien plus limitées à la fois en quantité et en qualité, puisque, si les surréalistes pratiquent parfois l'action directe, elles relèvent à peu près uniquement de la vie poétique, artistique ou intellectuelle. Il y a ainsi une profonde asymétrie d'inscription et de moyens dans la relation qu'entretiennent le Parti communiste français et le groupe surréaliste, asymétrie qui se traduit très concrètement par le fait que, si la question de l'adhésion au Parti communiste constitue un point central dans l'histoire du mouvement surréaliste, elle n'est en revanche 
qu'un détail dans l'histoire du Parti communiste et même un épisode secondaire dans l'histoire du rapport qu'il entretient avec l'ensemble des écrivains et des artistes. Breton est lucide quand il estime que le groupe est considéré comme « quantité négligeable ${ }^{1}$ » par le Parti. Et de fait : autant la question de la relation qu'il convient d'entretenir avec le Parti est envisagée avec gravité parce qu'elle menace la cohésion du groupe surréaliste et peut conduire à un éventuel éclatement, autant celle du ralliement éventuel des surréalistes n'a à peu près aucun impact sur l'histoire du Parti. Comme l'explique Jean-Pierre A. Bernard s'intéressant aux « rapports du P.C.F. avec les surréalistes », « l'ordre des facteurs est inversé et [...] il s'agit plutôt des rapports du surréalisme avec le communisme $»^{2}$. Et ce qui vaut pour les institutions trouve une transcription immédiate dans la vie des individus. Lorsque Breton adhère au Parti communiste au début de l'année 1926 et qu'on lui demande un rapport mobilisant des connaissances et des manières de penser qui ne sont pas les siennes, il réagit vivement : «On me demandait de faire à la cellule "du gaz" un rapport sur la situation italienne en spécifiant que je n'eusse à m'appuyer que sur des faits statistiques (production de l'acier, etc.) et surtout pas d'idéologie. Je n'ai pas $\mathrm{pu}^{3}$. » Le poète se récuse, il assiste à quelques réunions puis il prend ses distances, parce qu'il se sent méprisé comme poète et contraint de servir à contre-emploi. On imagine sans mal que, pour les autres adhérents de sa cellule et a fortiori pour l'ensemble des membres du Parti, son refus de rédiger un tel rapport, sa plainte écrite et son éloignement rapide de la base du Parti n'ont à peu près aucune répercussion.

L'asymétrie de puissance entre les deux regroupements ne doit toutefois pas cacher qu'ils ont tout deux en commun d'être des institutions définies par une forte contrainte collective mais également très hiérarchisées et traversées par de grandes tensions. L'un comme l'autre ne considèrent pas l'action, quelle que soit la manière dont ils l'envisagent, comme une catégorie d'abord individuelle. Ce n'est pas tel ou tel peintre ou poète qui doit servir dans son coin les buts que se donne le mouvement surréaliste, mais tous ses membres à l'unisson comme l'indique assez clairement le grand nombre de textes collectifs qu'ils signent, la référence récurrente chez les uns et les autres aux deux manifestes de Breton, l'emploi très fréquent du pronom nous dans les écrits de tel ou tel ou encore le titre d'une revue comme Le Surréalisme au service de la Révolution. Et de la même manière,

1. André Breton, «Légitime défense » [La Révolution surréaliste, n 8, décembre 1926], Point du jour [1934], repris dans Euvres complètes, t. II, Paris, Gallimard, coll. « Bibliothèque de la Pléiade », 1992, p. 286. La Révolution surréaliste sera désormais abrégé $L R S$. 2. Jean-Pierre Arthur Bernard, Le Parti communiste français et la question littéraire 1921-1939, Grenoble, Presses universitaires de Grenoble, 1972, p. 12.

3. André Breton, Second manifeste du surréalisme [LRS, no 12, décembre 1929], repris dans Euvres complètes, t. I, Paris, Gallimard, coll. « Bibliothèque de la Pléiade », 1988, p. $795-796$. 
du point de vue communiste, l'éclat de tel ou tel militant, aussi courageux ou brillant soit-il, compte moins que son obéissance. Cette ligne de conduite s'impose tout particulièrement à partir de la bolchevisation du Parti, dans les années 1923-1926. Comme on peut le lire dans L'Humanité à l'occasion de l'exclusion de Souvarine :

\begin{abstract}
$[\mathrm{L}] \mathrm{a}$ « valeur », le « talent », le « savoir » $[\ldots]$ de tel ou tel ne sauraient justifier un relâchement du contrôle auquel tous les communistes sont astreints. C'est dans la mesure où toutes les survivances petites-bourgeoises $\mathrm{du}$ «Moi » individualiste seront détruites, que se formera l'anonyme cohorte de fer des Bolcheviks français ${ }^{4}$.
\end{abstract}

Groupe surréaliste et Parti communiste sont tous deux des entités collectives définies par un projet commun et donc par une forme de discipline. Avec le premier, la poésie entre dans l'ère d'une contrainte de groupe autoproclamée qui passe par la mise en place et le respect de modalités nouvelles de création, souvent pratiquées en commun (récit de rêve, texte automatique, cadavre exquis, enquête, etc.); avec le second, la politique continue (après l'épisode matriciel de la guerre de 1914) de faire son entrée dans l'ère des masses qu'il convient d'organiser de manière rationnelle et sur la base d'une obéissance absolue.

Cette volonté d'une action collective parfaitement intégrée implique toutefois presque automatiquement son revers : le groupe surréaliste et le Parti communiste sont le lieu de rivalités permanentes. L'affirmation de cohésion doit faire la part des rapports de forces, des alliances et de leurs renversements, des renforcements et des affaiblissements de position, des départs et des exclusions, etc. Cet état de fait complique singulièrement l'étude de la relation entre le groupe surréaliste et le Parti communiste. Il n'y a pas seulement, d'un côté, le Parti communiste, de l'autre, le groupe surréaliste, mais deux institutions composées d'individus et d'institutions secondaires ou proches qui ne suivent pas nécessairement le même mouvement au même moment.

Concernant le champ communiste, on a mis en avant de manière très générale dans l'introduction de cet ouvrage les contradictions à l'œuvre, en France comme en URSS et entre la France et l'URSS, entre les différentes institutions qui, à des titres divers, revendiquent la direction de la politique artistique et littéraire communiste. Ces contradictions apparaissent clairement concernant la réception du surréalisme. Sur le fond d'une méfiance globale, il y a en effet place pour des considérations plus nuancées. À la fin de l'année 1925 et alors même qu'en France ils se sont rapprochés, non seulement du groupe Clarté, mais aussi du Parti lui-même en signant

4. L'Humanité (19 janvier 1924), cité par Nicole Racine et Louis Bodin, Le Parti communiste français pendant l'entre-deux-guerres [1972], Paris, Presses de la Fondation nationale des Sciences politiques, coll. « Références », 1982, p. 131. 
la déclaration collective « La Révolution d'abord et toujours! » qui paraît dans L'Humanité en septembre 1925, les surréalistes semblent inspirer une certaine sympathie au président du Bureau international de la littérature prolétarienne (BILP), Lounatcharski. Dans un article paru dans le même journal en décembre de la même année, ce dernier les complimente ainsi pour la tâche de dénonciation des valeurs bourgeoises qu'ils accomplissent et il les compare même aux écrivains prolétariens russes. Mais le ton change en grande partie à son retour en URSS, puisqu'en mars 1926, tout en faisant l'éloge d'Aragon, il dénonce leur subjectivisme, leur tendance anarchiste, les apparente cette fois aux avant-gardes expressionniste ou futuriste et se montre fort sceptique concernant leur rapprochement avec Clarté. La Société panrusse pour les relations culturelles avec l'étranger (VOKS) lui emboîte alors le pas mais elle s'attire imméditament les foudres de l'Association panunioniste des écrivains prolétariens (VAPP) qui, dans un geste de surenchère, lui reproche de faire trop de place aux surréalistes dont il ne faudrait même pas parler et à l'encontre desquels elle formule quant à elle un rejet total et méprisant, artistique aussi bien que politique. D'un point de vue tactique, Jean-Pierre Morel a raison de noter que, « dans cette querelle de boutiques, tout écrivain étranger qui attire l'attention d'un groupe russe est presque automatiquement discrédité aux yeux de l'autre groupe ${ }^{5} \gg$. Sur le fond, on peut aussi ajouter que ce n'est pas par hasard si ce sont le BILP et la VOKS qui montrent quelque mesure, tandis que la VAPP émet un jugement négatif tranché. Institution purement soviétique de promotion de la littérature prolétarienne, la VAPP entend faire des écrivains prolétariens soviétiques des modèles universels et mettre la littérature révolutionnaire mondiale à l'heure de l'URSS. Si le BILP est chargé de promouvoir la littérature prolétarienne dans le monde, il est aussi dans la nécessité de se ménager des alliés dans les différents pays et donc de laisser ouvertes des possibilités de collaboration. Quant à la VOKS, elle est animée par Olga Kameneva, femme de Kamenev et sœur de Trotski, qui s'est toujours opposée à l'idée d'une littérature prolétarienne spécifique et qui en outre, au cours de l'année 1926, se trouve peu à peu marginalisée par Staline.

Si elle est moins apparente parce qu'elle n'a que peu de traduction dans un organigramme institutionnel, une même division est à l'œuvre au sein du groupe surréaliste. Ses conséquences sont les mêmes que dans le Parti communiste français : blâme et rapide exclusion des fauteurs de trouble qui, de membres à part entière et parfois même jouant un rôle de premier plan, se trouvent brusquement rejetés hors du groupe. Notons toutefois que leur identification et leur sanction, si elles prennent un tour tout aussi théâtral qu'au Parti communiste, n'en connaissent pas moins une issue davantage prévisible. Le mouvement en effet est souverain, en ce sens qu'il ne dépend que de lui-même et qu'il n'est pas, de quelque manière que ce soit, soumis (1920-1932), Paris, Gallimard, coll. « Bibliothèque des idées », 1985, p. 104. 
aux décisions d'une autre entité. Bien plus, c'est lui qui occupe une position dominante dans le champ de l'avant-garde artistique et littéraire française des années 1920 et 1930. Du coup, ce qui régule les rapports de force qui se déroulent en son sein, c'est tout simplement le prestige de ses membres. La parole des fondateurs du mouvement passés par le groupe Dada et détenteurs déjà d'un certain capital symbolique par la qualité et le nombre de leurs publications a plus de poids que celle des membres arrivés plus récemment et aux publications moins nombreuses et moins prestigieuses. Au contraire de jeunes gens comme Marcel Noll ou Maxime Alexandre, d'autres - au premier rang desquels il faut citer Breton et Aragon - jouissent d'une autorité qui influe fortement sur les prises de positon collectives. Lorsque les différences s'exacerbent, il est toujours un moment où les membres les plus prestigieux du groupe le reprennent en main et font exclure ceux qui, d'après eux, menacent son identité ou sa cohérence. Au moment où le suicide semble fasciner un certain nombre des membres du mouvement, où l'influence paroxystique d'Artaud fait souffler un vent de folie, la réflexion sur une éventuelle adhésion au Parti communiste, qui s'enclenche à partir de l'été 1925, peut de ce point de vue paraître comme une manière pour Breton, non pas seulement d'assurer son pouvoir sur le groupe, mais aussi et plus simplement de lui permettre de survivre en l'orientant collectivement vers des considérations plus terre à terre. Libérer l'écriture de toutes les entraves de la raison ne va pas sans conséquence sur la raison elle-même. D'une certaine manière, il s'agit ici de se donner un cadre réflexif - et bientôt de se déterminer par rapport à une série de contraintes à la fois esthétiques et politiques - qui permette d'orienter l'inspiration, de la polariser, de la fixer, peut-être pour permettre aux poètes de se détourner au moins en partie et une partie du temps de la considération trop directe et brutale des motifs pulsionnels qui l'animent. Après la reprise en main de la direction de La Révolution surréaliste assumée par Breton en tête du numéro 4 de la revue en juillet 1925 qui lui permet de réduire l'influence métaphysique d'Artaud, on aboutit ainsi finalement à son exclusion en novembre de l'année suivante, parce qu'il refuse la politisation du mouvement et son rapprochement avec le Parti communiste.

Il faut ajouter que les rapports de groupe à groupe sont aussi toujours des rapports d'individus à individus plus ou moins formalisés. Parmi les membres du groupe Clarté, ils ne sont que trois à signer des articles personnels dans La Révolution surréaliste entre mars 1926 et décembre 1929 : Victor Crastre, Marcel Fourrier et Pierre Naville; les autres ne sont présents que par le biais des déclarations collectives. Inversement, entre novembre 1925 et mars 1927, ce sont toujours les mêmes noms surréalistes que l'on retrouve au sommaire de la revue Clarté : Aragon, Breton, Desnos, Éluard, Leiris et Péret. Comme le note justement Carole Reynaud Paligot, « certains surréalistes ont plus la tête politique que d'autres ${ }^{6} »$. Au cours des débats

6. Carole Reynaud Paligot, Parcours politique des surréalistes 1919-1969, Paris, CNRS Éditions, 1995, p. 2. 
qui animent le mouvement de l'été 1925 à la fin de l'année 1926, Artaud, Soupault et Desnos freinent le rapprochement avec le Parti communiste, tandis que Breton, Éluard, Péret ou Masson le favorisent et qu'Aragon peu à peu se rallie à leur position. De la même manière, certains communistes sont plus enclins que d'autres, soit du fait de leur fonction, soit par curiosité personnelle, à s'intéresser à la littérature et à l'art et, plus précisément encore, à favoriser une collaboration avec les surréalistes. Directeur des pages littéraires de L'Humanité de l'automne 1927 à septembre 1929, Georges Altman a beau à cette date avoir quitté le groupe Clarté, il ne s'en montre pas moins bien davantage favorable aux surréalistes que son successeur, du fait qu'il appartient à la même génération qu'eux et que, s'il n'a pas fait l'expérience de la guerre, leur sentiment de révolte lui est familier. Ce n'est pas parce que le Parti communiste ne prône pas officiellement un rapprochement avec les surréalistes que tel ou tel communiste ne fréquente pas tel ami poète; et inversement, il a pu arriver que tel surréaliste participe ponctuellement à telle action communiste sans que le groupe lui-même soit partie prenante. L'exemple de Naville le montre bien qui, d'abord membre du Parti communiste et du groupe surréaliste, insiste durant toute l'année 1926 auprès de ses amis poètes pour qu'ils rejoignent le Parti et abandonnent leur tendance anarchiste pour une attitude vraiment révolutionnaire, mais qui est lui-même exclu du Parti en mai 1928 pour déviance trotskiste.

L'existence de conflits internes entre institutions (et jusque chez les acteurs eux-mêmes) est ainsi une donnée qu'il ne faut pas oublier si l'on veut comprendre l'impression de grande confusion que donnent les dix années lors desquelles se pose la question de la relation entre le mouvement surréaliste et le Parti communiste. De l'été 1925, avec le rapprochement entre le groupe surréaliste et les membres du groupe Clarté, à l'été 1935, qui voit la publication de la déclaration collective « Du temps que les surréalistes avaient raison », c'est en effet un chassé-croisé incessant de projets communs et de collaborations avortées, d'enthousiasmes et de rebuffades, dans une relation qui hésite entre proximité et distance mais où se trouve toujours ménagé un espace de pensée et d'action commun, fût-il des plus ténus. Des individus et des institutions poussent dans le sens d'un rapprochement, tandis que d'autres favorisent au contraire un éloignement, voire une critique virulente, les uns et les autres pouvant même à l'occasion échanger leur position de manière apparemment incompréhensible mais en fait profondément liée à des changements de conjoncture politique. Reste que pour comprendre plus précisément la courbe globale et l'échec final de la relation entre surréalistes et communistes, il faut quitter les considérations institutionnelles et s'intéresser plus précisément à leur idéologie respective, aux stratégies et aux tactiques qu'ils mettent en œuvre.

D'un point de vue idéologique, la convergence semble d'abord évidente entre le mouvement surréaliste et le Parti communiste. Se définissant tous deux comme des avant-gardes, ils entendent en effet se 
placer dans le sens de l'histoire, là où le passé converge, où le présent se construit, où se prépare le futur. On ne peut ici que rappeler sommairement le point de vue communiste : renversement du système capitaliste et de la classe bourgeoise qui en tire profit, instauration d'une dictature transitoire du prolétariat, mise en place d'une société sans classe permettant la libre réalisation de chacun; et le point de vue surréaliste : révolte contre la société bourgeoise et la civilisation occidentale, rêve d'une poésie qui engloberait l'ensemble des manifestations de la vie humaine et permettrait à l'homme de libérer son désir. Ce qu'ont en commun communistes et surréalistes, c'est leur ennemi : la bourgeoisie, ses institutions et ses valeurs; et la même volonté de produire et diffuser une pensée critique, ainsi que de mener des actions qui ébranlent l'ordre en place. Comme leurs homologues du Parti communiste, les surréalistes font ainsi preuve d'une extrême virulence et ils s'élèvent contre « le petit système d'avilissement et de crétinisation en vigueur $^{7}$ ». Par la plume plus particulièrement violente de Crevel, Péret, Georges Sadoul ou Pierre Unik, ils mettent en cause, quand ils ne les couvrent pas d'injures, un certain nombre d'institutions comme la patrie, l'armée, la police, la justice, l'Église, l'Université ou l'hôpital psychiatrique. Ils dénoncent encore un certain nombre de pratiques sociales témoignant de l'avidité des uns et de l'abaissement moral des autres : la guerre, le sport de compétition, la colonisation ou le mariage. C'est en fait tout ce qui fonde la civilisation occidentale, la démocratie libérale et l'exploitation capitaliste qui se trouve passé au crible de la critique.

Sous ce rapport, un point est décisif : même si c'est dans des proportions variables, leur revendication de constituer l'avant-garde conduit surréalistes et communistes à occuper une position minoritaire dans leur champ respectif.

C'est vrai du mouvement surréaliste qui, même au moment de son plus grand rayonnement au début des années 1930, n'occupe pas la position dominante dans le champ littéraire, pas même dans le champ de production restreinte, que continuent de tenir les écrivains de la $N R F$. Cet état de fait a une conséquence très concrète : si certaines œuvres plastiques des artistes surréalistes se vendent bien, les chiffres de vente des poètes du groupe sont faibles. Quand ils ne sont pas soutenus par des parents ou quand ils n'exercent pas de profession rémunérée, ils connaissent ainsi une certaine précarité économique. Dès le numéro 2 de La Révolution surréaliste, Breton dénonce le « dénuement matériel » que subissent les grands poètes et qui constitue toujours une " entrave » à leur œuvre et il en vient à formuler des " revendications [...] sur le modèle des revendications ouvrières", c'est-à-dire à considérer comme indissociable la défense de « la liberté de pensée » et « celle d'exprimer cette pensée " ${ }^{8}$. Comme le souligne

7. André Breton, Second manifeste du surréalisme, op. cit., p. 783.

8. André Breton, « La dernière grève » [LRS, $\mathrm{n}^{\circ} 2$, janvier 1925], Euvres complètes, t. I, op. cit., p. 893. 
justement David Caute, « quand on parle de l'attraction exercée par le parti sur les intellectuels, il n'est naturellement par possible de négliger le facteur économique ${ }^{9}$ » et, en l'espèce, la précarité économique de nombre des membres du groupe surréaliste joue sans doute un rôle dans la proximité subjectivement éprouvée avec le prolétariat et dans l'attrait exercé conséquemment par le Parti communiste. Il faut ajouter que la critique extrêmement agressive que les surréalistes adressent aux valeurs, aux institutions et aux personnes accusées de faire le jeu de la bourgeoisie conduit à l'occasion à des procédures judiciaires, voire à de rapides interpellations. C'est le cas avec Sadoul, qui en juin 1930 a maille à partir avec la justice à la suite d'insultes et de menaces qu'il a proférées à l'encontre du major de Saint-Cyr ${ }^{10}$; le cas encore avec Aragon, inculpé en janvier 1932 après la publication du poème « Front rouge » appelant entre autres à faire :

Feu sur Léon Blum

Feu sur Boncour Frossard Déat

Feu sur les ours savants de la social-démocratie ${ }^{11}$.

Être un poète surréaliste ne va pas sans des prises de risque qui outrepassent assez largement la seule vie de l'esprit. C'est sans aucun doute pourquoi les réunions d'octobre et novembre 1925 entre surréalistes et membres du groupe Clarté sont marquées par une « atmosphère de clandestinité : engagement au secret, choix de pseudonymes, établissement d'un vocabulaire codé ${ }^{12} »$. Si la théâtralisation de l'action est indéniable, elle n'en repose pas moins sur quelques fondements.

Ce sentiment de danger et parfois ce danger réel qui valent pour les membres du groupe surréaliste valent a fortiori pour le Parti communiste. $\mathrm{Du}$ fait de sa nature révolutionnaire, ce parti politique n'a pas vocation à participer à un gouvernement ou même à soutenir son action; il ne le fait que de manière occasionnelle, au moment de compromis qu'il considère comme opportuns dans une certaine conjoncture. Concernant plus spécialement le Parti communiste des années 1920 et du début des années 1930, il faut en outre se défaire de la représentation du parti de masse tel qu'il apparaît au moment du Front populaire ou au sortir de la Seconde Guerre mondiale. Ce

9. David Caute, Le Communisme et les intellectuels français 1914-1966 [1964], Paris, Gallimard, coll. « La suite des temps », 1967, p. 38.

10. Voir les documents concernant l'affaire Sadoul/Keller, Le Surréalisme au service de la révolution, $\mathrm{n}^{\circ} 1$, juillet 1930, p. 34-40. Le Surréalisme au service de la révolution sera désormais abrégé $L S S R$.

11. Louis Aragon, «Front rouge » [Littérature de la Révolution mondiale, août 1931], Persécuteur persécuté [1931], repris dans Euvres poétiques complètes, t. I, Paris, Gallimard, coll. «Bibliothèque de la Pléiade », 2007, p. 496.

12. Vers l'action politique. De La Révolution d'abord et toujours! (juillet 1925) au projet de La Guerre civile (avril 1926), Présenté et annoté par Marguerite Bonnet, Paris, Gallimard, coll. "Archives du surréalisme », 1988, p. 11. 
parti perd des adhérents; à maints égards (en particulier par une certaine pratique du secret), il est organisé sur le modèle sectaire; il se situe en marge du jeu politique parlementaire et il subit les assauts des gouvernements successifs. On connaît la conclusion du discours prononcé par le ministre de 1'Intérieur Albert Sarraut à Constantine le 22 avril 1927 et dénonçant la politique antimilitariste et anticolonialiste du Parti communiste : « La destruction de la patrie n'est pas une opinion. C'est un crime. [...] le communisme, voilà l'ennemi ${ }^{13}$ ! » Comme le montre une célèbre affiche apparue dès 1919 , le communiste est alors perçu par les milieux dirigeants comme un homme au couteau entre les dents. Ce type de propos et de représentations a des conséquences très précises. Dans le contexte de la bolchevisation ordonnée par l'Internationale communiste dès la fin de 1923 et du tournant à gauche qui en constitue la conséquence logique (lancement de grèves, campagne contre la guerre du Rif, etc.), les poursuites et les condamnations d'élus communistes se multiplient. Avec l'adoption de la tactique « classe contre classe » en janvier 1928 par le Comité central, la chose empire encore : affrontements entre militants et policiers, arrestations préventives de responsables, inculpations qui touchent la presque totalité de la direction au nom d'un complot contre la sûreté intérieure et extérieure de l'État ou fermeture par la police des écoles de formation communistes. Alors au bord de la clandestinité, le Parti occupe une position à part et menacée dans le champ politique français.

D'un point de vue idéologique, surréalistes et communistes des années 1920 et du début des années 1930 se situent ainsi dans une rupture qui ne va pas sans danger et qui peut entraîner une précarisation des conditions d'existence, voire une sortie de la légalité. Il n'y a donc rien d'étonnant à ce que ce soit à l'occasion d'une critique contre la guerre du Maroc menée par le gouvernement français qu'ils opèrent une convergence plus précise. Dès l'été 1924, les communistes décident en effet de se mobiliser contre cette guerre coloniale et, le 10 septembre, Sémard, le tout nouveau secrétaire général du Parti, et le député Doriot envoient au nom du Parti communiste et des Jeunesses communistes un télégramme de félicitation à Abd el-Krim pour ses victoires contre les Espagnols. À partir de février 1925 suivent à la Chambre des députés une série de réquisitoires documentés, polémiques et virulents contre les présences impérialistes dans le royaume chérifien. La première déclaration conjointe des surréalistes et des membres des groupes Clarté et Philosophies prend place dans ce contexte. Parue dans L'Humanité le 2 juillet 1925 puis dans Clarté le 15 du même mois, elle reprend les principaux motifs de l'argumentaire communiste alliant antimilitarisme et refus de l'exploitation coloniale : dénonciation de l'usage des armes dans une guerre qui rappelle celle qui s'est déroulée il y a peu en Europe et remise en cause d'une prétendue œuvre de civilisation qui dissimule en fait une domination impérialiste. C'est ainsi d'abord sur le terrain strictement

13. Albert Sarraut, [Le Temps, 23 avril 1927], cité par Nicole Racine et Louis Bodin, op. cit., p. 152-153. 
politique (et non sur celui de l'art et de la littérature) que surréalistes et communistes ont engagé leur rapprochement.

Mais l'idéologie globalement partagée est une chose, et le rapport à l'idéologie en est une autre, qui se révèle être au moins aussi importante. Une avant-garde poétique comme le surréalisme et une avant-garde politique comme le Parti communiste sont des institutions totales. Le surréalisme n'a en effet pas pour vocation de révolutionner uniquement l'art ou la poésie mais l'ensemble de la vie dans toutes ses dimensions : morale, spirituelle, politique, sociale, etc; et de la même manière, le Parti communiste n'entend pas se cantonner à la sphère strictement politique et s'emparer du pouvoir dans le cadre prédéfini des institutions de la III ${ }^{e}$ République mais organiser aussi les sphères économique, juridique, artistique, littéraire, etc. dans un cadre politique complètement transformé (qui ne jouit du reste à ses yeux d'aucune autonomie réelle par rapport à l'infrastructure économique). La poésie n'est pas une fin pour les surréalistes; et la politique ne l'est pas davantage pour les communistes. De part et d'autre, il est question de rompre avec l'ancien monde et de créer une société et un homme nouveaux. On perçoit dès lors l'attrait que les deux partis peuvent exercer l'un sur l'autre mais aussi ce qui fonde pour la même raison l'impossibilité de fond d'un rapprochement durable.

Pour le surréalisme, recevoir le soutien du Parti communiste, c'est non seulement se lier avec le seul parti politique qui conteste avec la même radicalité que lui les valeurs et le fonctionnement de la société bourgeoise, mais encore et même plus garantir une diffusion dans la sphère politique de la pensée et des pratiques du mouvement, c'est-à-dire opérer une sortie du champ littéraire et s'imposer au-delà du petit cercle des initiés de l'avantgarde poétique. La chose est d'autant plus urgente que, dès l'entre-deuxguerres (et cela dure jusqu'aux années 1960), le Parti communiste fait peser une véritable tyrannie de l'action sur les milieux intellectuels et littéraires. Il n'est que de constater la défiance de Tzara à l'égard de « la poésie » :

Peut-on lui consacrer sa vie quand le moindre mouvement de rue, un peu plus vif que d'ordinaire, vous fait sursauter, vous fait croire que tout espoir n'est pas perdu? Agir, réellement agir! [...] La Révolution sociale n'a pas besoin de la poésie, mais cette dernière a besoin de la Révolution ${ }^{14}$.

Écrire et, en écrivant, agir : tel est le but des surréalistes qui ne peuvent se contenter, pour asseoir leur légitimité sous le rapport de l'action, des quelques coups de poings distribués par Aragon à tel critique malveillant avec Maïakovski ${ }^{15}$ ni même des souhaits de meurtre qui émaillent certains

14. Tristan Tzara, "Essai sur la situation de la poésie » [LSSR, n 4, décembre 1931], Euvres complètes, t. V, 1924-1963, Paris, Flammarion, 1982, p. 27-28.

15. «Un insulteur de Majakowsky reçoit une visite désagréable », $L S S R, n^{\circ} 1$, juillet 1930 , p. 21-22. 
articles, du type « À MORT MUSSOLINI! VIVE LA RÉVOLUTION MONDIALE ${ }^{16}$ ! ». Agir, pour les poètes du groupe, cela doit passer avant tout par l'écriture ou par l'œuvre d'art, et cela implique le relais d'une institution d'importance qui permette aux mots de pénétrer de nombreux esprits et d'actualiser leur puissance. Sous ce rapport, on peut affirmer que si les surréalistes rompent, au moins momentanément et collectivement, avec la mouvance anarchiste pourtant d'abord très influente au sein du mouvement, et s'ils se rapprochent du Parti communiste, c'est du fait d' " un désir d'efficacité ${ }^{17}$ » que seul ce dernier, appuyé de surcroît sur l'Union soviétique, est en mesure de satisfaire, parce qu'il constitue la seule force de contestation et la seule organisation révolutionnaire véritables, alors que le mouvement anarchiste est quant à lui dans les années 1920 en plein recul.

Pour le Parti communiste, gagner le groupe surréaliste, ce serait symétriquement étendre son empire au-delà du seul champ politique et imposer sa présence dans la sphère littéraire et artistique, ce qui, d'un point de vue très général, est également pour lui d'une extrême importance. Le Parti communiste a toujours essayé de rallier des artistes, des écrivains et des intellectuels. Même dans ses moments de grand isolement subi ou revendiqué, en particulier au moment de la stratégie « classe contre classe » adoptée par le Comité central en janvier 1928, il cherche à étendre sa sphère d'influence. Groupe surréaliste et Parti communiste, qui ont une même ambition révolutionnaire totalisante, ne peuvent ainsi que s'opposer. Pour les surréalistes, adhérer au Parti constitue en soi une forme d'héroïsme et dote le mouvement d'une dimension épique; pour le Parti communiste, l'héroïsme se définit à une autre échelle, dans une action qui, à l'imitation des révolutionnaires russes d'octobre 1917, doit conduire à la conquête du pouvoir. Sous ce rapport apparaît une contradiction insurmontable entre l'appartenance à l'avant-garde littéraire et 1'adhésion à l'avant-garde politique. D'un côté, on se trouve dans l'espace du champ littéraire qui tend à la plus grande autonomie des écrivains par rapport aux contraintes qu'imposent ou que cherchent à imposer les autres champs (politique et économique en particulier); de l'autre, on est, au contraire, dans l'espace du champ politique exigeant la plus grande soumission des écrivains à des impératifs proprement politiques. Pour qui entend être à la fois membre du groupe surréaliste et adhérent du Parti communiste, il y a ici une double injonction contradictoire, politique d'un côté, poétique et intellectuelle de l'autre, qui ne peut a priori se résoudre en un éthos commun ou simplement de compromis.

La première incidence de cette injonction contradictoire est d'ordre strictement politique. Sur le fond, là où le Parti communiste exige une

16. Paul Éluard et Benjamin Péret, « Revue de la presse » [LRS, no 8, décembre 1928], repris dans Benjamin Péret, Euvres complètes, t. VII, Paris, Librairie José Corti, 1995, p. 22.

17. Carole Reynaud Paligot, op. cit., p. 36. 
fidélité absolue et un respect sans faille du principe du centralisme démocratique, les surréalistes ne renoncent jamais à leur liberté de jugement. La déclaration collective signée avec Clarté advient ainsi un an après la mise en branle de la campagne politique contre la guerre du Maroc orchestrée par le Parti communiste. En d'autres termes : c'est le Parti qui a pris l'initiative, et les surréalistes qui ont suivi. Pour le Parti communiste, la possibilité d'un mouvement inverse relève tout simplement de l'impensable. Ce n'est pas seulement que le Parti comme institution de contrainte collective se méfie de toute action individuelle qui pourrait lui échapper; c'est plus encore qu'il entend promouvoir l'apparition de ce que David Caute nomme « un corps cohérent et discipliné d'intellectuels fidèles ${ }^{18} \gg$ et qu'il met en place au fil des ans un appareil institutionnel de formation et de contrôle susceptible de lui permettre d'atteindre cet objectif. Or, la revendication d'une autonomie intellectuelle et d'un droit de regard politique est un véritable leitmotiv du groupe surréaliste, qu'on retrouve dans chacun des textes d'importance qui scandent l'histoire de ses rapports avec le Parti communiste. Les surréalistes ne sauraient être les simples porte-paroles de pensées ou les simples relais de modalités d'action élaborées ailleurs et sans eux. L'éthos proprement intellectuel des membres du mouvement, qui implique non seulement de participer au débat, mais surtout de le faire vivre sans cesse, s'oppose ici à l'éthos militant, qui pousse au contraire à faire siens les points de vue et les décisions de la hiérarchie et qui ne va pas sans un certain anti-intellectualisme. Les surréalistes citent souvent les autorités philosophiques et politiques que sont Hegel, Feuerbach, Marx, Engels ou Lénine; ils publient des extraits de leurs œuvres ou de textes qui leur sont consacrés; ils s'appuient sur eux pour envisager l'actualité politique et, d'un point de vue plus théorique, ils se réclament du matérialisme historique et de la dialectique. Mais s'ils donnent des gages de leur conversion au marxisme et de leur engagement en faveur de la révolution russe, ils refusent toujours qu'une position leur soit autoritairement imposée. De la dénonciation encore ponctuelle du journal L'Humanité que dirige alors Barbusse comme publication " puérile, déclamatoire, inutilement crétinisante », en particulier parce qu'elle se fait le relais d' « une méthode de propagande [...] déplorable » excluant « les courants véritables de la pensée moderne » et favorisant l'obéissance aux « autorités », à la stigmatisation plus générale du " besoin frénétique d'orthodoxie » et d' « une politique de compromis de plus en plus graves $»{ }^{19}$ poussant l'URSS à soutenir la politique de la France impérialiste, à se montrer réactionnaire en matière de mœurs, d'art et de littérature et à imposer un culte du chef dangereux pour la révolution, les conséquences de cette revendication sont une critique incessante qui ne fait que s'amplifier avec les années. De ce point de vue, il n'y a

18. David Caute, op. cit., p. 17.

19. André Breton, «Légitime défense », op. cit., p. 283-285, et « Du temps que les surréalistes avaient raison » [août 1935], ibid., respectivement p. 464 et p. 466. 
rien d'étonnant à voir les surréalistes se rapprocher du Parti communiste par le biais d'une relation avec la revue Clarté dont les membres sont comme eux des intellectuels occupant une position relativement marginale au sein de la mouvance révolutionnaire. Le devenir des membres du groupe Clarté, qui dès le mois de décembre 1925 basculent dans le soutien à un Trotski en voie de marginalisation en URSS, ne fait du reste et fort logiquement qu'anticiper celui de nombre de surréalistes qui se rapprochent plus tard d'un trotskisme minoritaire et tolérant mieux le débat qu'un Parti communiste en voie de stalinisation.

Le rapport différent à la vie intellectuelle que présentent poètes surréalistes et militants communistes entraîne le cas échéant des divergences de fond; il entraîne aussi un rapport différent à la stratégie. En se rapprochant du Parti communiste, les surréalistes n'ont pas abandonné la révolte héritée des années dada; ils ont juste accepté qu'en certaines occasions elle se canalise dans l'action commune et concertée. Quand Aragon ou Desnos en appellent en un crescendo graphique à « la Terreur » et à «LA TERREUR » et quand le second exige même qu'on remette « la guillotine » à l'honneur afin d'engager « l'épuration méthodique de la population $»^{20}$, cela ne peut apparaître aux yeux des membres du Parti que comme un appel prématuré à l'action et plus sûrement encore comme du nihilisme petit-bourgeois. Pour les surréalistes, il s'agit au contraire d'une affirmation puissamment créatrice qui active la crise de conscience et participe de cette liquidation des coordonnées spirituelles et mentales du temps. Un parti politique comptant a minima quelques dizaines de milliers de membres ne peut toutefois fonctionner comme un groupe poétique de quelques dizaines d'individus. En lieu et place d'un éthos artiste de la provocation jetée à la face du pouvoir politique (mais les surréalistes n'ambitionnent jamais de l'occuper), les communistes opposent un éthos de la responsabilité qui exige le respect d'un principe d'obéissance (préparant l'exercice du pouvoir). Le premier est pertinent dans le cadre d'une stratégie d'occupation de l'espace avantgardiste du champ littéraire fondé sur le principe de « l'institutionnalisation de la révolution permanente ${ }^{21} »$; le second, dans une stratégie qui concerne l'organisation d'un renversement du champ politique. On comprend dès lors pourquoi un article du 21 septembre 1925 paru dans L'Humanité salue les poètes surréalistes comme de jeunes gens « d'extraction bourgeoise pour la plupart, [...] venus d'instinct à la Révolution » mais précise pour finir que « la foi révolutionnaire doit être raisonnée, systématique, elle doit s'étayer sur les lois économiques généralement formulées par Marx et par

20. Respectivement Louis Aragon, «Communisme et Révolution » [LRS, n 2, janvier 1925], Chroniques 1918-1932, Paris, Stock, 1998, p. 227, et Robert Desnos, « Description d'une Révolte prochaine » [LRS, n 3, avril 1926], Euvres, Paris, Gallimard, coll. « Quarto », 1999, p. 253.

21. Pierre Bourdieu, Les Règles de l'art. Genèse et structure du champ littéraire, Paris, Seuil, coll. «Libre Examen », 1992, p. 305. 
Lénine $»^{22}$. La leçon prime sur le compliment, et l'appel à l'esprit d'orthodoxie s'impose en lieu et place d'une révolte perçue comme incontrôlable et donc nuisible à la cause révolutionnaire.

On touche ici à la seconde conséquence de l'injonction contradictoire venue des champs poétique et politique, qui est d'ordre essentiellement esthétique. À aucun moment Breton et ses amis ne consentent à renoncer à l'autonomie de la poésie et de l'art, tandis que le Parti communiste, de son côté, revendique leur contrôle.

Du côté des surréalistes, il n'est que de considérer la position qu'adopte Breton à la suite de l'inculpation d'Aragon par la justice française pour la publication de " Front ronge ». Ce poème relève à ses yeux d'une esthétique de la propagande qu'il réprouve mais, en même temps, il tient à le défendre : «Nous nous élevons contre toute tentation d'interprétation d'un texte poétique à des fins judiciaires et réclamons la cessation immédiate des poursuites ${ }^{23}$. » Alors que, du côté communiste, l'honneur de tout militant est de répondre de ses actes, d'en assumer les éventuelles conséquences pénales au point qu'un séjour en prison est perçu comme une forme d'initiation, du côté surréaliste, on affirme une liberté absolue de la poésie qui n'a de compte à rendre ni devant la justice des hommes ni devant telle ou telle instance politique. Cette défense de Breton repose sur une position de principe déjà énoncée dans le Second manifeste du surréalisme, lorsqu'il fait l'éloge de " ces produits de l'activité psychique, aussi distraits que possible de la volonté de signifier, aussi allégés que possible des idées de responsabilité toujours prêtes à agir comme freins, aussi indépendants que possible de tout ce qui n'est pas la vie passive de l'intelligence » que sont «l'écriture automatique et les récits de rêve ${ }^{24}$. Il y a plus ici qu'une éventuelle divergence idéologique placée sur le seul plan intellectuel : une nouvelle différence d'éthos entre l'affirmation des droits de la subjectivité poétique et sa négation juridique ou politique. Non pas telle ou telle pensée, mais le rapport à la pensée et à la créativité : poésie ne s'autorisant que d'elle-même, de son propre mouvement et de sa propre histoire $v s$ littérature, sinon systématiquement de propagande, au moins soumise à un contrôle collectif dont les objectifs ne sont pas proprement littéraires mais relèvent d'autres sphères de l'activité sociale. Or, aux yeux des surréalistes, en aucun cas l'activité critique du mouvement ne doit supplanter sa libre expérimentation. Comme l'écrit Aragon, « les hommes ont tout à apprendre des poètes, et non pas des poètes en tant qu'hommes mais des poètes en tant que poètes. Les poètes tiennent du sismographe plus que du citoyen ${ }^{25} \gg$.

22. Carole Reynaud Paligot, op. cit., p. 56.

23. André Breton, Misère de la poésie [1932], repris dans Euvres complètes, t. II, op. cit., p. 5 .

24. André Breton, Second manifeste du surréalisme, op. cit., p. 809-810.

25. Louis Aragon, «Introduction à $1930 »\left[L R S, \mathrm{n}^{\circ} 12\right.$, décembre 1930], Chroniques 1918-1932, op. cit., p. 341. 
Aussi capricieuse puisse-t-elle sembler (mais les surréalistes sont persuadés que, dans le fond, elle ne l'est pas), la subjectivité ne se négocie pas, elle est souveraine, car elle se confond avec l'énergie poétique elle-même et avec sa puissance de révélation et de transformation du monde.

À s'en tenir à ces principes, le Parti n'entend pas faire du groupe surréaliste - ni d'ailleurs de n'importe quel autre groupe - le dépositaire de sa politique artistique et littéraire. Même lors de la période où leur proximité est la plus grande, entre la fin de l'année 1926 et la fin de l'année 1930, les porte-paroles officiels de sa politique en matière culturelle entendent en effet la garder sous leur contrôle. Pour la période 1920-1932, Jean-Pierre Morel est sans aucun doute celui qui a étudié avec le plus de précision la mise en place d'institutions soviétiques et internationales permettant le passage de l'idéologie à l'organisation, $i$. e. d'une production littéraire relevant de la superstructure à la fabrication d'une arme mise au service du prolétariat (ou de ses représentants officiels). Dès le début des années 1920 et jusqu'au tournant de 1933, un anti-intellectualisme certain pousse à marginaliser les auteurs bourgeois, à utiliser les petits-bourgeois comme force d'appoint, mais en les surveillant de près, et surtout à donner aux écrivains prolétariens la haute main sur la littérature révolutionnaire. «Pour la première fois, peut-être, dans l'histoire, un mouvement littéraire fait dépendre la création de l'organisation ${ }^{26}$. » Même si dans les instances du Parti communiste français et jusque très tardivement, la différence n' est pas toujours bien faite entre auteurs prolétariens et compagnons de route (alors que cette opposition déchire le champ littéraire russe), même si les institutions officielles peuvent prendre des positions pas toujours accordées et laissent ainsi une certaine marge de manœuvre aux écrivains, il n'en reste pas moins que le désir de contrôle demeure présent. De son point de vue qui privilégie l'autonomie du poétique sur le politique, Breton a raison de se plaindre que le militant Michel Marty adresse à l'un de ses amis cette remontrance : « Si vous êtes marxiste [...], vous n'avez pas besoin d'être surréaliste ${ }^{27}$. » Mais Marty exprime clairement ce qu'il en est de la position du parti, pour lequel être marxiste (et plus encore léniniste) subsume tout autre type d'identité et conduit à une identification complète au Parti. Le refus des surréalistes de prendre au sérieux toute forme de littérature prolétarienne actuelle et leur revendication d'une esthétique qui leur soit propre inspire la méfiance, voire l'hostilité des cadres littéraires communistes - rappelant ainsi l'attitude plus que réservée de Lénine devant l'œuvre de Maïakovski. Avec la mise en avant de la littérature prolétarienne, il s'agit de faire passer « les intérêts du militantisme avant ceux du surréalisme » et d'orchestrer donc ce que Jean-Pierre Morel nomme une «"tchékisation" de la littérature ${ }^{28}$.

26. Jean-Pierre Morel, op. cit., p. 54.

27. André Breton, Second manifeste du surréalisme, op. cit., p. 795.

28. Jean-Pierre Morel, op. cit., respectivement p. 381 et p. 384. 
Face à l'instauration de ce contrôle, Breton tente de dialectiser le rapport entre action poétique et action politique - la première gardant une légitime autonomie tant que la seconde exige un certain attentisme du fait d'une conjoncture défavorable à toute prise de pouvoir. Récusant en théorie comme en pratique « l'opposition de la réalité intérieure au monde des faits », il explique ainsi dès Légitime défense en décembre 1926 - et il ne varie pas sur ce point :

Dans le domaine des faits, de notre part, aucune équivoque n'est possible : il n'est personne de nous qui ne souhaite le passage du pouvoir des mains de la bourgeoisie à celles du prolétariat. En attendant, il n'en est pas moins nécessaire, selon nous, que les expériences de la vie intérieure se poursuivent et cela, bien entendu, sans contrôle extérieur, même marxiste ${ }^{29}$.

Que cet intérêt maintenu pour la vie intérieure conduise les surréalistes à lire les ouvrages du comte Hermann von Keyserling ou ceux de Freud, qu'il conduise à une valorisation (éphémère) de l'Orient ou à un intérêt (plus durable) pour la psychanalyse, il s'agit à chaque fois de trouver des ressources intellectuelles pour affirmer, d'un point de vue théorique mais aussi dans la conjoncture présente, l'autonomie de la poésie et le refus d'un arraisonnement prématuré à une littérature définie comme prolétarienne. Aucun doute : "L'imaginaire est ce qui tend à devenir réel ${ }^{30}$ ", et Breton affirme là une fonction, non pas utopique de la poésie, mais créatrice au sens le plus fort du terme, complétant ici et maintenant, à sa manière, sur un plan poétique et moral, l'œuvre plus lointaine de la révolution politique proprement dite. Certes, une large partie des centres d'intérêt du surréalisme semble échapper totalement à l'injonction politique et à la pensée marxiste. Il en est ainsi de l'intérêt pour le rêve, pour la voyance, pour la pensée ésotérique, pour la psychanalyse ou pour la peinture. De manière exemplaire, le thème de l'amour demeure omniprésent, associé de surcroît à un intérêt marqué pour une sexualité que marquent des motifs comme l'onanisme, le fétichisme, la sodomie, le sadisme ou le masochisme. Mais que ce soit par les textes prenant pour objet la femme, les recherches sur la sexualité de janvier 1928, l'enquête sur l'amour de décembre 1929 ou encore la défense de la figure sulfureuse de Sade dans Le Surréalisme au service de la Révolution, les surréalistes mettent en avant la libération d'un désir qui, loin d'être ce qu'un Parti communiste familialiste et puritain voit en lui, à savoir le témoignage des perversions d'intellectuels décadents, constitue à leurs yeux une véritable force révolutionnaire. De Desnos déclarant que « l'héroïsme désormais se [confond] avec l'amour » à Char expliquant se mouvoir « dans un paysage où la Révolution et l'Amour

29. André Breton, « Légitime défense », op. cit., p. 292.

30. André Breton, «Il y aura une fois » [LSSR, $\mathrm{n}^{\circ} 1$, juillet 1930], Le Revolver à cheveux blancs [1932], repris dans CEuvres complètes, t. II, op. cit., p. 50. 
allument, de concert, d'étonnantes perspectives ${ }^{31}$, le désir et la révolution vont ensemble, et d'abord, dans le meilleur des cas : font ensemble poème. Contre le couple des amants refermé sur lui-même, contre toute barrière qui séparerait la vie privée du désir et la vie publique des intérêts politiques, au plus haut degré d'intégration de son engagement révolutionnaire et de sa vocation poétique, le surréalisme ne sépare pas vie intérieure et vie extérieure et il affirme une convergence des libérations et des luttes qui préfigure à bien des égards le freudo-marxisme (théorie) et la révolte étudiante de Mai 68 (pratique).

À ces positions de principe concernant l'autonomie des prises de positon intellectuelles et des pratiques esthétiques s'ajoutent pour finir des considérations stratégiques et tactiques qui ont à voir avec la manière dont communistes et surréalistes entendent très concrètement briser leur isolement respectif du fait de leur position avant-gardiste. Le Parti communiste dispose sous ce rapport d'un choix bien plus large au sein du champ littéraire que le surréalisme au sein du champ politique. Avant même de considérer ce qu'il implique précisément en termes esthétique et politique, le choix d'une alliance privilégiée avec le groupe surréaliste ne constitue pas une priorité pour le Parti, parce qu'il s'agit d'un ensemble d'écrivains qui n'occupent pas une position dominante dans le champ littéraire. C'est en effet une constante de la stratégie du Parti communiste qui, du début des années 1920 à la fin des années 1930 (et même au-delà), préfère toujours, soit susciter des écrivains et des intellectuels dans ses rangs, soit favoriser le ralliement ou la sympathie de personnalités déjà bien reconnues. La première stratégie est celle des rabcors (ou correspondants ouvriers) de la littérature prolétarienne; la seconde, celle des compagnons de route ou des adhérents célèbres. Aux surréalistes peu ou mal connus du grand public et au statut symbolique encore incertain, le Parti préfère des écrivains comme France, Barbusse ou Rolland qui assurent une visibilité plus grande de ses idées et témoignent mieux de son influence. Mieux encore, il n'est pas rare qu'il loue des auteurs comme Claudel ou Cocteau qui ne sont pourtant pas de son bord politique mais en lesquels il reconnaît la grandeur de la tradition littéraire nationale. Ce type de positions réconciliatrices revendiqué essentiellement par Monde dans l'entre-deux-guerres devient même après la Seconde Guerre mondiale un élément central de la politique culturelle officielle du Parti. Or, ce choix communiste de la valorisation des écrivains détenteurs d'un fort capital symbolique constitue un motif majeur de brouille pour les surréalistes. Ne concevant pas la poésie de la même manière que la grande majorité des autres poètes et faisant reposer leur légitimité sur une rupture avec la tradition et une recomposition du canon des grands textes, ils engagent de violentes polémiques contre telle Euvres complètes, Paris, Gallimard, coll. « Bibliothèque de la Pléiade », 1983, p. 1235. 
ou telle grande figure du monde des lettres (Barrès, France ou Claudel). Pour célébrer comme il se doit la mort de France, les surréalistes publient ainsi le tract « Un cadavre » qui paraît le 14 octobre 1924. Rien de tel du côté communiste : «l'admiration pour Anatole France est alors en Russie la chose la mieux partagée ${ }^{32}$ »et, à Paris, L'Humanité a rendu hommage à l'écrivain ami de la Russie soviétique dans son numéro du 13 avril 1924 à l'occasion de ses quatre-vingts ans. Les surréalistes, qui s'en tiennent aux principes et ne peuvent dissocier révolutions esthétique et politique, ne peuvent tolérer que les journaux et revues du Parti fassent preuve de réticence à leur encontre et accréditent au contraire des écrivains qu'ils considèrent comme réactionnaires à tous égards.

Autre point stratégique important, le Parti cherche à s'adjoindre, non pas des groupes, qui, dotés d'une identité et d'une solidarité collectives, seraient susceptibles d'opposer une résistance à ses propres organes de décision et de contrôle, mais des individus isolés et donc beaucoup plus faciles à circonscrire. Telle est sans aucun doute la cause profonde de l'échec de la création de La Guerre civile, la revue que les surréalistes et les membres du groupe Clarté envisagent de fonder en fusionnant leur revue respective et qui devait paraître au printemps 1926. Si elle ne voit pas le jour, c'est certes aussi, comme l'explique Marguerite Bonnet, du fait de « la difficulté de faire cohabiter dans un ensemble cohérent des esprits aux préoccupations et aux visées si différentes ${ }^{33} »$. Mais quand Breton explique que les uns et les autres se sont heurtés à « la crainte d'aller contre les desseins véritables de l'Internationale communiste ${ }^{34} »$, il pointe aussi des raisons externes, et en particulier l'inquiétude du Parti communiste, comme l'explique Marion, membre du Bureau politique, de voir « un groupuscule ", « une petite chapelle » ou encore " une petite école » revendiquer « le point de vue du Parti ${ }^{35}$. Toujours fidèles à leur tactique de captation individuelle, les autorités du Parti communiste entreprennent plus tard de semer la division dans le groupe surréaliste en sommant tel ou tel membre de clarifier sa position. Le meilleur exemple de ce processus est bien sûr l'affaire Aragon, qui, à son retour de Kharkov en décembre 1930, se trouve pris dans un conflit de légitimité entre les déclarations critiques à l'encontre du surréalisme qu'il a accepté de signer en Russie et les protestations de fidélité au mouvement qu'il profère par la suite et qui, mis devant l'incompatibilité de ses deux appartenances, choisit finalement en mars 1932 de rompre avec le surréalisme et d'affirmer sa solidarité avec le Parti.

La stratégie du surréalisme est a contrario de se faire reconnaître, et par les plus hautes instances dirigeantes, non seulement comme groupe, ce

32. Jean-Pierre Morel, op. cit., p. 88.

33. Vers l'action politique, op. cit., p. 15.

34. André Breton, "Légitime défense », op. cit., p. 295.

35. Cité dans Vers l'action politique, op. cit., p. 18. 
qui d'un point de vue organisationnel garantit la préservation d'une force de résistance collective, mais plus encore, d'un point de vue strictement poétique, comme le groupe légitime - seule manière de garantir l'alliance théorico-pratique des avant-gardismes poétique et politique, ainsi que d'assurer au mouvement une position hégémonique sur l'ensemble de la production esthétique révolutionnaire. Si les surréalistes n'ont ainsi de cesse de critiquer les auteurs bourgeois, leurs cibles privilégiées sont surtout les auteurs qui, comme eux, font assaut de déclarations révolutionnaires mais développent leur œuvre dans un cadre esthétique qu'ils jugent réactionnaire ou conservateur. Telle est sans aucun doute la raison pour laquelle, plus encore que Barrès ou Claudel, ce sont des sympathisants comme France, Berl ou Gide et, plus encore, un membre du Parti comme Barbusse, qui attirent leurs attaques les plus violentes. Cette hostilité n'est pas un fait de conjoncture. Elle est manifeste dès l'offensive que lance Clarté en février 1926, elle se poursuit avec la publication de Légitime défense par Breton en décembre 1926 et dans un nouveau numéro de Clarté en mars 1927. Après Aragon, qui considère Monde comme « une ordure confusionnelle, qui associe à toute propagande prosoviétique dosée tout un peuple de chiens, de traîtres et de littérateurs ${ }^{36} »$, nombre de surréalistes reprennent cette critique qui vise l'écrivain et l'intellectuel discrédités qu'est à leurs yeux l'auteur du $\mathrm{Feu}$, les uns, à l'occasion du débat sur la littérature prolétarienne organisé par la revue en décembre 1930, les autres, l'année suivante à l'occasion de la publication dans les pages de sa revue d'un éloge de Claudel ou de Noailles.

Pour comprendre l'histoire de la relation entre communistes et surréalistes, reste l'impact de la conjoncture qui est finalement décisive en ce qu'elle implique des changements tactiques qui font pencher chacun des deux groupes, tantôt du côté de l'alliance intéressée, tantôt de la prise de distance, tantôt et pour finir de la rupture. Il y a un type de conjonctures favorable au rapprochement, et un autre type qui ne l'est pas : dans les moments de faiblesse partagée (qui sont aussi des moments où l'unité cède la place à des sécessions et à des exclusions), surréalistes et communistes ont tout intérêt à se rapprocher; dans des moments où le plus puissant des deux s'estime en position de force, il peut se permettre d'exercer une pression sur l'autre pour le plier à ses propres impératifs - ce qui finit par conduire immanquablement à la rupture du lien et à une dislocation au moins relative du groupe le plus faible.

À partir de ces considérations, il est possible de scander en quatre étapes l'histoire des relations entre le groupe surréaliste et le Parti communiste : 1) un rapprochement progressif entre l'été 1925 (début de la collaboration des surréalistes avec le groupe Clarté) et le courant de l'année 1927 (adhésion de Péret, Aragon, Breton, Éluard et Unik au Parti communiste fin 1926 pour

36. Louis Aragon, "Monde, Samedi 23 novembre » [LRS, n 12, décembre 1929], Chroniques 1918-1932, op. cit., p. 337. 
le premier et début 1927 pour les autres), au moment où le Parti communiste est à son point le plus bas du point de vue électoral et très isolé sur la scène intellectuelle, alors que de son côté le surréalisme traverse une crise interne majeure; 2) entre 1928 et la fin 1930, une prise de distance informelle (les cinq ne renouvellent pas leur adhésion en 1928), lors de laquelle les deux parties constatent très concrètement que le rapprochement est impossible mais ont encore besoin l'une de l'autre et se gardent donc d'engager les hostilités, du fait de leur faiblesse respective, le Parti communiste parce qu'il a lancé la tactique « classe contre classe » qui contribue à l'isoler encore davantage, le groupe surréaliste parce qu'il a fort affaire avec le groupe rival du Grand Jeu et que son exigence de politisation lui permet très concrètement de le faire éclater; 3 ) à partir du Congrès de Kharkov en novembre 1930, un moment d'affrontement, lorsque, la position politique du Parti communiste se renforçant d'un point de vue électoral et dans la sphère intellectuelle et littéraire, il tente de faire plier le surréalisme en lui imposant de respecter sa ligne politique et ses principes esthétiques; 4) la rupture le 10 mars 1932, lorsque le Parti se trouve en mesure de diviser le groupe surréaliste en affirmant dans L'Humanité qu'il a gagné Aragon à sa cause (suivent Maxime Alexandre, Georges Sadoul et Pierre Unik) et que le groupe surréaliste comprend qu'il n'a rien à gagner à une telle alliance qui lui ferait perdre son autonomie et sa spécificité.

Contrairement à ce que l'on aurait pu penser, l'abandon de la tactique « classe contre classe » qui intervient au courant de l'année 1934 et qui se traduit dans le domaine littéraire par la dissolution de la RAPP et la mise en place d'une politique d'ouverture à l'égard des écrivains sympathisants du Parti ne constitue pas une circonstance favorable pour une alliance avec les surréalistes. Certes, elle rend les instances du Parti communiste français bien plus tolérantes d'un point de vue esthétique et même politique et elle serait donc susceptible de raviver le lien entre surréalistes et communistes, mais elle interdit dans le même temps et contradictoirement l'établissement d'un lien privilégié - seul acceptable du point de vue surréaliste - entre le mouvement et le Parti. À la tutelle esthétique et politique honnie (parce qu'elle nie l'autonomie du champ littéraire dans son ensemble) succède un consensus qui n'est pas davantage désirable (parce qu'il ne correspond pas à la radicalité de cet espace particulier du champ littéraire qu'est l'avant-garde). Le but des différentes institutions culturelles qui se mettent en place et qui, à leur façon, contribuent au regain de popularité du Parti dans l'opinion et au triomphe du Front populaire au printemps 1936, n'est en effet pas de confier la politique littéraire et plus largement culturelle du Parti à un groupe d'avant-garde, important certes dans sa sphère propre, mais bien trop limité dans son rayonnement pour correspondre à un grand mouvement de masse. Le Parti s'ouvre, mais il préfère s'en remettre - plus encore que d'habitude - à des auteurs dont la carrière est largement faite et qui mettent leur notoriété au service de l'action commune des partis de 
gauche, quand bien même, à l'exception de Barbusse et de Rolland, ils n'auraient jamais, pas toujours ou pas depuis longtemps donné les gages de leur engagement révolutionnaire. Tel est le but de la fondation de l'Association des écrivains et artistes révolutionnaires (AÉAR), dont l'assemblée constitutive se tient le 17 mars 1933 et dont les surréalistes sont tenus à distance avant d'en être exclus et de n'y faire plus que de fugaces apparitions jusqu'en juin 1935 .

On voit avec cet exemple de la relation entre surréalistes et communistes que c'est bien d' ' une aliénation mutuelle des avant-gardes politique et culturelle ${ }^{37}$ » qu'il faut parler. Après avoir affirmé que " l'homologie entre les positions dans le champ littéraire (etc.) et les positions dans le champ social global n'est jamais aussi parfaite que celle qui s'établit entre le champ littéraire et le champ du pouvoir où se recrute, la plupart du temps, l'essentiel de sa clientèle », Bourdieu précise dans Les Règles de l'art que, si « les écrivains et les artistes qui sont situés au pôle économiquement dominé (et symboliquement dominant) du champ littéraire, lui-même temporellement dominé, peuvent [...] se sentir solidaires (au moins dans leurs refus et leurs révoltes) des occupants des positions dominées, économiquement et culturellement, dans l'espace social », « ces alliances » ne sont pas « exemptes de malentendus, voire d'une sorte de mauvaise foi structurale $»^{38}$. Et il ajoute dans une note :

[C] ette utopie [...] se heurte sans cesse à l'évidence de la difficulté pratique
de surmonter, autrement que dans les impostures ostentatoires du radical
chic, l'écart structural, malgré l'homologie, entre les positions « avancées »
dans le champ politique et dans le champ artistique et, du même coup, le
décalage, voire la contradiction, entre le raffinement esthétique et le pro-
gressisme politique ${ }^{39}$.

$\mathrm{Si}$, avec le Parti communiste et le groupe surréaliste, on a bien un exemple parfait de correspondance entre institutions qui sont situées dans le même espace de leur champ respectif - l'espace de l'avant-garde - et qui toutefois ne sont pas en mesure de converger dans des pratiques et des discours communs, il convient toutefois, plutôt que de blâmer les surréalistes d'un point de vue moral - ce que Bourdieu laisse entendre avec les notions de " mauvaise foi » et d' " impostures ostentatoires »-, de considérer les logiques semblables et différentes qui sont à l'œuvre ici et là. Groupe surréaliste et Parti communiste se ressemblent à maints égards : par leur organisation collective fortement contraignante, par leur position relativement marginale dans leur champ respectif et par leur positionnement idéologique exigeant une transformation totale de l'homme et de la

37. David Caute, op. cit., p. 114.

38. Pierre Bourdieu, op. cit., p. 349-350.

39. Ibid., note 57 , p. 350. 
société. Mais ces trois points communs par lesquels se définit une position d'avant-garde ne peuvent suffire à assurer un rapprochement durable, dans la mesure où ils reposent sur des légitimités sociales et des éthos individuels étrangers les uns aux autres, dépendant, ici, du champ littéraire, et, là, du champ politique : ni les modalités de la prise de position politique, ni la constitution d'une esthétique révolutionnaire, ni même les considérations stratégiques et tactiques ne peuvent s'entendre de part et d'autre de la même manière. Les avant-gardes surréaliste et communiste - et il faudrait considérer plus largement à leur lumière ce qu'il en est des avant-gardes littéraires et politiques au $\mathrm{Xx}^{\mathrm{e}}$ siècle - ne peuvent rester indifférentes l'une à l'autre mais elles ne peuvent non plus converger. Produites par une même société en crise mais situées dans des champs différents, elles rêvent chacune de leur côté à une humanité réconciliée, tout en continuant de porter en elles et jusque dans leur relation l'héritage des divisions sociales. " "Transformer le monde", a dit Marx; "Changer la vie", a dit Rimbaud ${ }^{40} »$ : contrairement à ce qu'espérait Breton, ces deux mots d'ordre lancés, pour l'un, à partir de la philosophie et de la politique, pour l'autre, à partir de la poésie, n'en firent un que de manière très occasionnelle.

Guillaume Bridet Université Paris 13 - CENEL 